Article,

\title{
New Business and Operating Models. Optimization of a blast furnace in the steel industry. Machine Learning as a Process Optimization.
}

\author{
Andrés Redchuk ${ }^{1}$, Federico Walas Mateo ${ }^{2 *}$
}

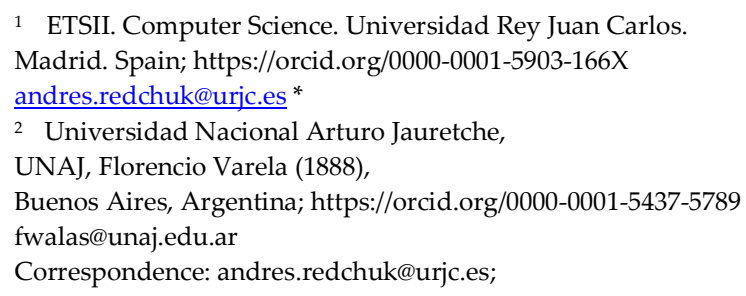

\begin{abstract}
Background: The article takes the case of the adoption of machine learning in a steel manufacturing process through a platform provided by a novel Canadian startup, Canvass Analytics. This way the steel company could optimize the process in a blast furnace. The content of the paper includes a conceptual framework on key factors around steel manufacturing and machine learning. Method: The article takes the case of the adoption of machine learning in a steel manufacturing process through a platform provided by a novel Canadian startup, Canvass Analytics. This way the steel company could optimize the process in a blast furnace. The content of the paper includes a conceptual framework on key factors around steel manufacturing and machine learning. Results: This case is relevant for the authors by the way the business model proposed by the startup attempts to democratize Artificial Intelligence and Machine Learning in industrial environments. This way the startup delivers value to facilitate traditional industries to obtain better operational results, and contribute to a better use of resources. Conclusion: This work is focused on opportunities that arise around Artificial Intelligence as a driver for new business and operating models. Besides the paper looks into the framework of the adoption of Artificial Intelligence and Machine Learning in a traditional industrial environment towards a smart manufacturing approach.
\end{abstract}

Keywords: Artificial Intelligence; Machine Learning; Smart manufacturing; Industry 4.0; business model; operating model

\section{Introduction}

This paper pretends to visualize how artificial intelligence (AI) facilitates creating new business models and how manufacturing can achieve competitive advantage through new operating models. The work from [1] highlights the disruptive potential of $\mathrm{AI}$ and more recently machine learning (ML) to generate new business models and the opportunities for entrepreneurs. The authors support the conceptual development with many cases from practice. They conclude that the innovative potential of the new business can give rise radical new operating models that could lead to firms of a kind not seen before.

Another point to attend regarding the content of this article is raised by [2] regarding opportunities for the development of smart industries, and its expansion and evolution of production processes. This is a result of the demand for more efficient technologies and procedures, quality standards and cost reduction, and also by technological improvement. Furthermore, a large variety of issues are discussed on Industry 4.0, the main focus of the new production paradigm is to bring into existing industries more intelligent and adaptable processes, with better use of production resources. In the article the authors give an interesting approach to industrial process optimization and smart manufacturing.

A key issue to consider is that AI need high amount of data to deliver value results. [3] consider big data as a high impact tool for potentially important economic and social 
value and for gaining competitive advantage. In this article it is affirmed that big data has grown from a $\$ 6.8$ billion industry to $\$ 32$ billion industry only in three years and forecasts the market of big data technology and services to grow at a 23.1 percent compound annual rate, reaching $\$ 48.6$ billion in 2019 . Therefore with the volumes of organizational data moving past terabytes to tens or even hundreds of petabytes, businesses and information technology (IT) leaders are embracing unique opportunities to capitalize on big data to gain the competitive advantage. It is also considered in this article that companies are spending more than 10 percent of the IT budget on data and are considering big data and analytics as a strategic asset to support their decision-making and improve business processes.

To complete the idea brought in the previous paragraphs, [4] highlights that there is a huge quantity of legacy, enterprise and operational systems data that is not being used. The article points that manufacturers are sitting on a goldmine of unexplored historical, legacy and operational data from their Manufacturing Execution Systems (MES), Enterprise Resource Planning systems (ERP), among other software sources, and they cannot afford to miss out on its unexplored potential. However, only $20-30 \%$ of the value from such available data-at-rest is currently accrued.

An interesting article about the evolution of Artificial intelligence (AI) is brought by [5]. In this work he states that analytics 4.0 is the next step in analytical sophistication for organizations, and it is the era of artificial intelligence or cognitive technologies. It had been widely adopted with rates, depending upon geography, of 20 to $30 \%$ across large enterprises in 2016 and 2017. It features not only the use of AI methods, but also greater use of autonomy in the execution of the methods, particularly automated machine learning (ML).

Another issue to address is the definition of business operating model and business model. [6] Define that the value of a firm is shaped by these two concepts. The firm's business model, defined as the way the firm promises to create and capture value. The firm's operating model, defined as the way the firm delivers the value to its customers.

This article considers the ideas in the previous paragraphs and presents a case of adoption of $\mathrm{AI} / \mathrm{ML}$ to optimize the process at a blast furnace of a steel company. The work aims to go deeper from previous work regarding new business and operating models based on AI/ML solutions under Industry 4.0 framework, following [7], and the impact of the novel business model in complex industrial processes to boost operative performance.

The case is focused in the adoption of a new operating model under a ML solution through an AI platform offered by the startup Canvass Analytics to improve steel production processes. The paper intends to illustrate the way new business models based on $\mathrm{AI} / \mathrm{ML}$ platforms transforms traditional industrial processes and takes them to new levels of competitiveness [8]. The value proposal of the new business model is focused in easing the implementation process in the industrial environment.

The work begins with a conceptual framework to describe the key concepts involved in the case reviewing works on $\mathrm{AI} / \mathrm{ML}$, and some key concepts about data analysis related to steel industry. Then introduces the startup and its business model, to finally describe the case illustrating the new operating model, the solution adopted and the results achieved.

\section{Related Works and conceptual framework in steelmaking}

There are a number of works regarding ML in the steel industry, a simple query with ML and steel industry as key words at Scopus scientific data base, performed on august 27, 2021, brought 99 works regarding the subject.

Among the most interesting ones to consider related to the case studied are the works of [9], [10], [11], [12], [13], and [14].

From the works mentioned in the above paragraph the one from [9] acquires a great relevance for the case is presented in this paper, due to the fact that referrers to ML based computational methodology used for improvement in the productivity of the blast furnace at Jindal Steel and Power Limited (JSPL), India. These improvements took about 
eighteen months and were made possible by, among other things, the use of ML employed alongside domain knowledge of blast furnace specialists.

The authors highlight the importance of ML methods in improving productivity and quality in continuous flow materials processing, such as in a blast furnace that takes in various earth ore materials and outputs hot metal.

According to [10] a structured Business Intelligence platform is the basis for the use of a statistical data analytics approach, empowered by emerging technologies of AI and ML for the extraction of hidden knowledge useful for production, cost and quality optimization. The steelmaking industry is an open field to apply these techniques: the high availability of data and the necessity to control complex and multi-physics processes meet the requirements for a successful implementation of a data-driven approach. They show this at ABS, Acciaierie Bertoli Safau, Italy, where together with the expertise of industrial partners, started using Predictive Analytics.

Another interesting work is presented by [11] in which presents a methodology to contribute with solutions for facing the challenges of data processing and rendering, and predictive model design, which have been deployed in a factory of ACERINOX Europa S.A.U.

The paper of [12] at Gerdau Aços Longos SA, Brazil, brings a solution based on ML to make possible to have a billet with a steel grade different than expected misplaced and loaded. The methodology identifies the steel of the bar being rolled through the rolling stands motors current usage. Using an outlier detection technique, it was possible to identify patterns in the data for every steel grade, identifying when it did not match the expected in simulated data. Currently the model is working offline, and further testing will be realized during production for validating the model efficiency.

[13] Presents a case of Falkonry's Operational AI, as a novel solution to approach the challenges of scaling machine learning and $\mathrm{AI}$ in manufacturing. This intelligence first step to creating machine learning applications is based on a consistent, repeatable, time series classification technique robust to incomplete and irregular data, engenders expert knowledge capture, and leads to real-time actions to positively affect production. The paper discuss underlying challenges in the real time application of Operational AI to production systems, and presents a real-world case study and how Operational AI is applied in steel manufacturing at production scale to predict equipment failure to reduce unexpected downtime

Finally [14] address the difficulty to apply AI and ML in traditional industries, mainly for two reasons: (a) It isn't really big data, although Megabytes are being logged by the comprehensive automation system of steel plants and from thousands of field sensors every week and (b) purely data-based model results have a likelihood of some 70 or $80 \%$ to deliver the correct result or decision proposal, but this won't deliver an assured best/optimized control mode for production or quality. Up to now such likelihood percentage doesn't seem sufficient in the Business to Business (B2B) field.

The last two papers are particularly interesting for this work because both referrers to business models on AI/ML to facilitate the adoption of analytics solutions in traditional industries like steel production.

\subsection{Artificial Intelligence and Machine Learning (AI/ML) and Traditional Data Analytics}

An article by [15] affirm that process systems in steelmaking are complex entities, involving raw materials, products, energy, processes, automation systems and people. Their efficient operation involves numerous functions and tasks. A commonly used classification for process systems includes Planning, Scheduling, Real-time optimization, and Control.

More specifically [9] points that modern blast furnace $(\mathrm{BF})$ processes are influenced by many factors, quite a few of them interacting with each other. It is fair to say that the process is immensely complex. While process complexity in modern state of the art 
blast furnaces have the positive effect of allowing a higher theoretical limit on both productivity and quality, but this also lends itself to undesirable chronic patterns of behaviour that become intractable, leading to lower productivity and quality. It is in this context that machine learning methods become a useful device and a vehicle for discovery.

The complexity of the processes at the blast furnace for steel smelting can be seen in the work of [16]. Where it is shown the mathematical model for blast furnaces. A threedimensional unsteady state model to express the operation behaviour of a blast furnace, and understand the complexity of the process inside the furnace.

The ML model learns the dynamics of the process to make predictions to anticipate anomalies or incidents that affect the optimization of the process. [17] Show that ML adapts better to changes in processes and facilitates proactivity. The ML model learns from data to change conditions faster and keep model predictions consistent and stable, while traditional solutions require a lot of adjustments or more rules and are generally reactive.

ML develop accurate models for a variety of complex problems [18]. In the case of the Blast Furnace, very complex chemical and thermodynamic issues arise. ML models for solutions to a wide variety of problems and fit for greater precision with algorithms.

\section{The approach with CANVASS ANALYTICS Platform}

[7] Refers to the business model of Canvass Analytics [19]. Gradient Ventures is Google's AI-focused venture fund [20] which invest in and connect early-stage startups with resources in artificial intelligence. It is interesting to search in the portfolio of startups founded, and found Canvass Analytics as one of them. This startup that aroused interest for this research had developed a business model based on an AI-powered predictive analytics platform for Industrial processes. Its customers include leading manufacturing and energy companies globally.

[7] Mention the startup Canvass Analytics as a leading provider of AI industrial software. Its patent-pending technology enables industrial companies to accelerate their digitization strategy by putting AI directly in the hands of plant operators - empowering them with data-driven insights to improve production processes and optimize assets.

Unlike other solutions, Canvass AI empowers process engineers to quickly apply AI data applications without requiring coding skills. The solution offers prebuilt templates to minimize coding for process engineers and business analysts. By operationalizing machine learning, operators use Canvass AI to improve Overall Equipment Effectiveness (OEE) by addressing industrial manufacturing's most important AI use cases, such as anomaly detection, asset and process optimization, defect part detection, asset failure prediction, forecasting, and to model what-if scenarios. Across the world, Fortune 5000 companies use Canvass AI to reduce greenhouse gas emissions and operating costs each year by optimizing production processes and assets, improving quality, and reducing waste.

At its web site the startup says that its platform automates the entire data science process, eliminating consulting data science projects. They affirm that the platform approach has accelerated the time to insights 12 times faster than other solutions and approaches. The solution is developed specifically for the industrial sector.

Canvass Analytics provide a Solution as a Service (SaaS) ingesting in its platform, which is cloud hosted, data in data series format from MES or IIoT solutions. The AI/ML platform is integrated with the shop floor solutions, and work with data in real time to give the predictions. A complex issue for the industrial environment, and a weakness reported by the paper of [21] like cybersecurity, is tackled by hosting the platform at Azure Microsoft Cloud. This cloud solution also gives the calculation power for the requirement of $\mathrm{AI} / \mathrm{ML}$ models.

[7] points that the startup provides a solution that automates the entire data science process, eliminating consulting data science projects. Canvass business model has accelerated the time to insights 12 times faster than other solutions and approaches. The solution is developed specifically for the industrial sector. 
Canvass AI is being applied across the production process to improve quality, reducing energy consumption, and reducing waste in raw material processing, steelmaking and casting, and hot rolling. That has led to greater stability downstream in rolling processes, leading to more plant throughput, fast sales cycles and less deterioration of its refractories. In addition, Canvass AI is being used to control co-generation boilers to optimize fuel source and consumption, optimize energy supply to plant demand, and reduce energy waste and fuel costs [22].

\section{Adoption case in the Steel Industry}

The case examined is the process at the blast furnace of Stelco [23], a Canadian Steel company, where there was a need to optimize the production line improving the quality and quantity of performance at the blast furnace. As mention before these are complex and dynamic processes that generate hundreds of data and the variability is high. This leads to management challenges to maintain consistency of production quality.

It should be considered that inconsistencies in quality in the casting process, generates excess scrap, rework, production delays, among others. Traditional quality control was done through samples that were analysed in a laboratory. The problem with this methodology is that if there was an inconsistency, the problem was solved in a reactive way, when it had already occurred.

On the other hand, it must be considered that the process runs in three eight hour shifts, and the operators are different, each with their own criteria and experience on the process. In addition, the smelting process includes a large number of chemical and thermodynamic variables that are very difficult to monitor and control.

The firm generates real time data from the production processes through a world class MES software, OSI PI from OSIsoft [24]. This data is stored in historian type databases.

\subsection{Solution and results}

To address the improvements in the process, Stelco chose the solution proposed by Canvass AI with the following objectives:

1-Identify the parameters that influence the maximization of production performance using process data generated by the OSI PI platform.

2-Predict product quality and performance at different intervals and use those predictions to adjust control parameters in real time, thus maximizing product quality and performance.

3-Apply a proactive policy for the resolution of problems and anomalies of the process based on having advance information about what happens in the casting process.

4-Institutionalize the best practices of the operators and provide those responsible for the information processes that facilitate decision-making.

The solution was adopted after identifying project objectives mentioned above. Then the historical data was revised according quantity and quality for the model to learn from. Then and exploration and preparation phase was performed to get insight from data and prepare for modelling. The last step with the historical data was to train the model and validate results. 
Then the in house historian data base from OSI PI was integrated through an application programming interface (API). This way the data from the processes is ingested into Canvass AI platform that is hosted at the Azure cloud, in real time streaming. The model was productionized and then real time predictions began to appear. The results were monitored, and adjustments performed. Finally the model results give predictions to facilitate deciding changes in the process for its improvement. Figure 1 shows the seven step process followed to achieve results that allow optimize the processes.

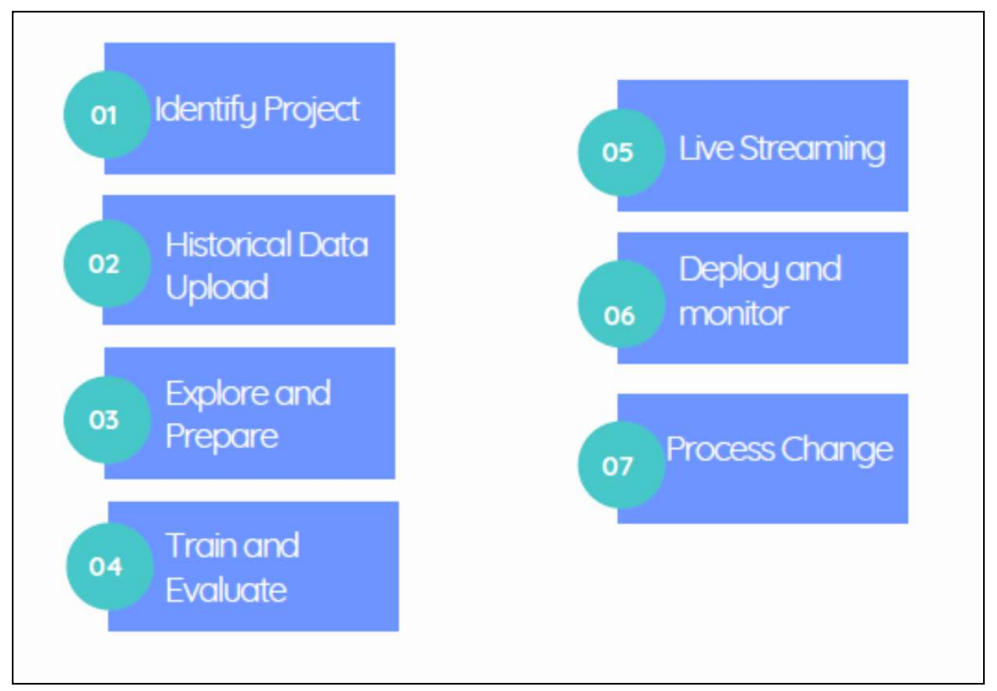

Figure 1. Seven step process followed at Stelco to have results with CANVASS AI platform.

Canvass AI platform made it easy to define the relationship between variables that significantly affect the production result, like quantity of silicon added, and fuel consumption. AI models are collaborating to determine which of the control variables have a positive or negative impact on production quality. By applying a ML reinforcement learning the result of the production is predicted in certain duly specified intervals.

Historical data were taken from a period of one year, and parameters like energy consumption, temperature, silicon content, among others, were analysed to generate a model that allows predicting when the alloy with the highest silicon content should be conditioned and when to increase or decrease the oven temperature. In this way, it works proactively and the operator has advance information for decision-making.

By using Canvass AI platform with process data from the blast furnace, it is possible to predict future production and control quality. In addition, the operator from the control room can virtually adjust control variables and see potential changes in real time in the production process.

As a result of applying AI through various operations in steel production, the client has obtained a competitive advantage by improving processes, and achieving consistency in quality with silicon content always at a normal range. Besides by defining set points in real time for fuel consumption, facilitating energy savings. It also reduced the percentage of scrap, reducing costs and producing higher quality steel. 
The use of ML methods employed alongside domain knowledge of blast furnace specialists, facilitate a greater knowledge of the process which is augmented continuously. That allows working on permanent improvement and achieving better Key Performance Indicators (KPI).

\section{Discussion}

The case approaches the adoption of a new operating model based on AI/ML in a traditional industrial environment, as the steel production process. The solution came from a startup that get advantage from new methodologies that shorten times and simplify adoption of analytics in the industry, and offer a new business model driven by this methodology.

An important observation deserves change management with the new business model, which generates results faster and with lower complexity than traditional analytics solutions. This is because the model prosed by Canvass is centred in the end user, the process operator. The model from the Canadian Startup eases consuming and risky tasks linked with software development and algorithms.

The new operating model is based on predictive analytics aiming at exploiting the huge treasure of legacy operational data and to overcome some challenges of real-time data analytics. The potential of the proposed approach is high in traditional industries that have not benefit from the advancements of Industry 4.0. In most cases have just started investigating the potential of data analytics and machine learning for the optimization of their production processes.

Another point to highlight, is meant by the opportunities that the smart manufacturing paradigm opens to startups that develops solutions to ease the adoption of AI/ML models to the industry, and the way they could shorten implementation cycles from several months, like the case of Jindal Steel and Power Limited, to a couple of months as shown in the case at Stelco.

In line with the above paragraph is the solution presented by Falkonry's Operational $\mathrm{AI}$ at section 2 of the work to improve equipment maintenance. The case of Falkonry's could be studied thoroughly to broaden the concepts on this paper and it is considered for next steps in this research.

Future work could also be related to research on cases of business models on AI/ML applied in traditional industrial sectors that need to optimize the use of resources to go towards a net zero emissions target like energy, and oil \& gas, among other.

Author Contributions: Conceptualization, methodology, formal analysis, investigation, and writing - review and editing, Walas Mateo, Federico. Resources.; visualization; supervision.; project administration, and funding acquisition, Redchuk, Andres. All authors have read and agreed to the published version of the manuscript.

Funding: "This research received no external funding"

\section{Acknowledgment}

The authors wish to thank two research projects that made possible the content of this article. The projects are "Metodologías de abordaje al modelo Industria 4.0 en PyMEs, el rol de la Empresas de Base Tecnológica (EBT), los recursos humanos, y el ecosistema de 
Innovación", approved by Resolution (R) No. 183-21- UNAJ INVESTIGA Program, and "Mejora de Procesos, Optimización y Data Analytics: Aplicación en Procesos e Industrias de Interés Regional Mediante Estudios de Casos Reales", approved by Resolution of the UNLZ Engineering Faculty.

\section{References}

1. Pfau, W., Rimpp, P. (2021). “AI-Enhanced Business Models”. Digital Entrepreneurship, Digital Entrepreneurship, Future of Business and Finance,Springer International Publishing. https://doi.org/10.1007/978-3-030-53914-6_7.

2. Da Silva, VL., Kovaleski, JL, Negri Pagani, R, De Matos Silva, J, Corsi, A. (2019). Implementation of Industry 4.0 concept in companies: empirical evidences, International Journal of Computer Integrated Manufacturing.

3. Grover, V., Chiang, RHL., Liang, T.-P., Zhang, D. (2018). Creating Strategic Business Value from Big Data Analytics: A Research Framework, Journal of $\quad$ Management Information Systems, 35:2, 388-423, DOI: $\quad$ 10.1080/07421222.2018.1451951

4. Lepenioti, K., Pertselakis, M., Bousdekis, A., Fenareti Lampathaki, A. L., Apostolou, D., Mentzas, G., Anastasiou. S. (2020) “Machine Learning for Predictive and Prescriptive Analytics of Operational Data in Smart Manufacturing." Dupuy-Chessa S., Proper H. (eds) Advanced Information Systems Engineering Workshops. CAiSE 2020. Lecture Notes in Business Information Processing, vol 382. Springer, Cham. https://doi.org/10.1007/978-3-030-49165-9 1

5. Davenport, T. H. (2018). "From analytics to artificial intelligence", Journal of Business Analytics, 1:2, 73-80, DOI: 10.1080/2573234X.2018.1543535.

6. Iansiti, M., Lakhani. D. (2020). Competing in the age of AI: strategy and leadership when algorithms and networks run the world / Boston, MA: Harvard Business Review Press.

7. Walas Mateo, F., Redchuk, A. (2021). “The Emergence of New Business and Operating Models under the Industrial Digital Paradigm. Industrial Internet of Things, Platforms, and Artificial Intelligence/Machine Learning". Volume 11, Number 2, 2021 of JMEA. USA. ISSN 2159-5283

8. Walas Mateo, F. (2020). "Industry 4.0 and the Emergence of New Business Models." Presented at the Eighteen LACCEI International Multi-conference for Engineering, Education and Technology, Buenos Aires, Argentina.

9. Mishra, R., Gandhi, A., Jaiswal, R., Mishra, S., Fadnis, A. (2020) Machine Learning to Improve Blast Furnace Productivity at Jindal Steel \& Power. AISTech 2020 - Proceedings of the Iron \& Steel Technology Conference DOI 10.33313/380/029.

10. Spadaccini, A., Di Pierro, M., Nadalutti, P., Cestari, L., Pellegrini, G.. (2018). Data-driven prediction models at Acciaierie bertoli safau meltshop. ICS 2018 - 7th International Congress on Science and Technology of Steelmaking: The Challenge of Industry 4.0.

11. Ruiz-Sarmiento, J.R., Monroy, J., Moreno, F.A., Galindo, C., Bonelo, J. M, Gonzalez-Jimenez, J. (2020). A predictive model for the maintenance of industrial machinery in the con-text of industry 4.0. Engineering Applications of Artificial Intelligence 87 103289

12. Gazola, P. E., dos Santos, H. S. O., Daroit, M., Locatelli, A.. (2021). A Machine Learning Approach For Steel Grade Detection in Hot Rolling Mills AISTech 2021 - Proceedings of the Iron \& Steel Technology Conference 29 June-1 July 2021, Nashville, Tenn., USA DOI 10.33313/382/18

13. Waters, C., Klemme, B., Talla, R., Jain, P., Mehta, N. (2021) Transforming Metal Production by Maximizing Revenue Generation With Operational AI AISTech 2021 - Proceedings of the Iron \& Steel Technology Conference 29 June-1 July 2021, Nashville, Tenn., USA DOI 10.33313/382/115

14. Strasser, S., Hohenbichler, G., Sattler, M., Krahwinkler, P., Reidetschlaeger. J. (2020). Artificial Intelligence Services in Steel Production - On Premises and in the Cloud. AISTech 2020 - Proceedings of the Iron \& Steel Technology Conference DOI 10.33313/380/209

15. Daoutidis, P., Lee, J.H., Harjunkoski, I., Skogestad, S., Baldea, M., and Georgakis, C. (2018). Integrating operations and control: A perspective and roadmap for future research. Computers \& Chemical Engineering, Vol. 115, pp. 179-184.

16. Nishioka, K., Ujisawa, Y., Akatani, K. (2018) Development of Mathematical Models for Blast Furnaces. NIPPON STEEL \& SUMITOMO METAL TECHNICAL REPORT No. 120 December 2018 UDC 669. 162. 263: 681. 3 Technical Report.

17. Azeem, M., Haleem, A., and Javaid, M.. (2021). “Symbiotic Relationship between Machine Learning and Industry 4.0: A Review". Journal of Industrial Integration and Management. Vol. 6, No. 2 1-33.World Scientific Publishing Co. DOI: $10.1142 /$ S24248622213000271.

18. Jamwal, A., Agrawal, R., Sharma, M., Kumar, A., Kumar, V., and Garza-Reyes, J.A.A. (2021), "Machine learning applications for sustainable manufacturing: a bibliometric-based review for future research", Journal of Enterprise Information Management, Vol. ahead-of-print No. ahead-of-print. https://doi.org/10.1108/JEIM-09-2020-0361.

19. Canvass Analytics website, 2021. http://www.canvass.ai, [Online; Accessed 26 July 2021].

20. Gradient Ventures website, 2021. https://www.gradient.com/, [Online; Accessed 26 July 2021].

21. Davenport, T. H. (2020). Is AI Getting easier? Forbes Media LLC.

22. Steel Market Magazine, May 2020 edition. https://www.amm.com/Issue/101498/May\%202020.html

23. Stelco website, 2021 www.stelco.com [Online; Accessed 26 July 2021].

24. OSIsoft website, 2021 www.osisoft.com [Online; Accessed 26 July 2021]. 